\title{
Relativistic Study of Spinless Particles for Generalized Hylleraas Potential with Position Dependent Mass
}

\author{
Akaninyene D. Antia and Eno E. Ituen \\ Department of Physics, University of Uyo, P.M.B. 1017, Uyo, Akwa Ibom State, 520271, Nigeria
}

\begin{abstract}
The relativistic study of spinless particles under a special case of equal scalar and vector generalized Hylleraas potential with position dependent mass has been studied. The energy eigenvalues and the corresponding wave functions expressed in terms of a Jacobi polynomial are obtained using the parametric generalization of NU (Nikiforo-Uvarov) method. In obtaining the solutions for this system, we have used an approximation scheme to evaluate the centrifugal term (potential barrier). To test the accuracy of the result, we compared the approximation scheme with the centrifugal term and the result shows a good agreement with the centrifugal term for a short-range potential. The results obtained in this work would have many applications in semiconductor quantum well structures, quantum dots, quantum liquids. Under limiting cases, the results could be used to study the binding energy and interaction of some diatomic molecules which is of great applications in nuclear physics, atomic and molecular physics and other related areas. We have also discussed few special cases of generalized Hylleraas potential such as Rosen-Morse, Woods-Saxon and Hulthen potentials.
\end{abstract}

Key words: Relativistic Klein-Gordon equation, generalized Hylleraas potential, position dependent mass, parametric Nikiforov-Uvarov method, centrifugal term.

\section{Introduction}

Studies of exactly solvable potentials have attracted much attention since the early development of quantum mechanics [1-3] and obtaining solutions for the nonrelativistic and relativistic equations for some potentials of interest is still an interesting work in the existing literature [4-13]. In nuclear and high energy physics, one of the interesting problems is to obtain exact solution of the Klein-Gordon, Duffin-Kemmer-Petiau and Dirac equations. When a particle is in a strong potential field, the relativistic effect must be considered, which gives the correction for nonrelativistic quantum mechanics [14, 15].

In nonrelativistic quantum mechanics, it is well known that the exact solutions of Schrödinger equation are possible only for a few set of quantum systems. However, when arbitrary angular momentum quantum number $l$ is present, one can only solve the Schrödinger equation approximately using suitable

Corresponding author: Akaninyene Daniel Antia, Ph.D., lecturer, research fields: theoretical physics, mathematical physics, quantum mechanics and computational physics. approximation schemes [16]. Some of such approximations include conventional approximation scheme proposed by Greene and Aldrich [17], improved approximation scheme by Jia et al. [18], elegant approximation scheme [19] etc. These approximations are used to deal with the centrifugal term or potential barrier arising from the problem.

In solving nonrelativistic or relativistic wave equation whether for central or noncentral potential, various methods are used. These methods include AIM (Asymptotic iteration method) [20], SUSYQM (Super symmetric quantum mechanics) [21], shifted $\frac{1}{N}$ expression [22], factorization method [23, 24], NU (Nikiforov-Uvarov) method [25] and others [26, 27]. In the relativistic quantum mechanics, one can apply the Klein-Gordon equation to the treatment of a zero-spin particle. In recent years, many studies have been carried out to explore the relativistic energy eigenvalues and corresponding wave functions of the Klein-Gordon and Dirac equations [14, 15, 28]. 
The aim of this paper is to apply the parametric generalization of NU method to study the relativistic spinless particles under equal scalar and vector generalized Hylleraas potential with position dependent mass. The GHP (generalized Hylleraas potential) is defined as [29-31]:

$$
V(r)=V_{1} \frac{a+e^{\alpha r}}{b+e^{\alpha r}}-V_{2} \frac{d+e^{\alpha r}}{b+e^{\alpha r}},
$$

where, $a(\neq b), \quad b$ and $d(\neq b)$ are the Hylleraas parameters and $\alpha$ is the range of the potential. $V_{1}, V_{2}$ are potential depths, and this potential could be used to describe nucleon-nucleon interactions, meson-meson interaction and also in various branches of nuclear physics and quantum chemistry.

\section{Generalized Parametric (Nikiforov-Uvarov) Method}

The NU method was presented by Nikiforov and Uvarov [25] and has been employed to solve second order differential equations such as the (SWE Schrödinger wave equation), KGE (Klein-Gordon equation), DE (Dirac equation), etc. The SWE:

$$
\psi^{\prime \prime}(r)+[E-V(r)] \psi(r)=0
$$

which can be solved by transforming it into a hypergeometric type equation through using the transformation, $s=s(x)$ and its resulting equation is expressed as:

$$
\psi^{\prime \prime}(s)+\frac{\bar{\tau}(s)}{\sigma(s)} \psi^{\prime}(s)+\frac{\tilde{\sigma}(s)}{\sigma^{2}(s)} \psi(s)=0
$$

where, $\sigma(s)$ and $\tilde{\sigma}(s)$ must be polynomials of at most second degree and $\bar{\tau}(s)$ is a polynomial with at most first degree and $\psi(s)$ is a function of the hypergeometric type.

The parametric generalization of the NU method is given by the generalized hypergeometirc-type equation as [32]:

$$
\begin{gathered}
\psi^{\prime \prime}(s)+\frac{\left(c_{1}-c_{2} s\right)}{s\left(1-c_{3} s\right)} \psi^{\prime}(s)+ \\
\frac{1}{s^{2}\left(1-c_{3} s\right)^{2}}\left[-\xi_{1} s^{2}+\xi_{2} s-\xi_{3}\right] \psi(s)=0 .
\end{gathered}
$$

Eq. (4) is solved by comparing it with Eq. (3), and the following polynomials are obtained:

$$
\begin{gathered}
\tilde{\tau}(s)=\left(c_{1}-c_{2} s\right), \sigma(s)= \\
s\left(1-c_{3} s\right), \tilde{\sigma}(s)=-\xi_{1} s^{2}+\xi_{2} s-\xi_{3} .
\end{gathered}
$$

According to the NU method, the energy eigenvalues equation and eigen functions, respectively, satisfy the following sets of equation

$$
\begin{gathered}
c_{2} n-(2 n+1) c_{5}+(2 n+1)\left(\sqrt{c_{9}}+c_{3} \sqrt{c_{8}}\right)+ \\
n(n-1) c_{3}+c_{7}+2 c_{3} c_{8}+2 \sqrt{c_{8} c_{9}}=0, \\
\psi(s)=. \\
N_{n l} S^{c_{12}}\left(1-c_{3} s\right)^{-c_{12}-\left(c_{13} / c_{3}\right)} P_{n}^{\left(c_{10}-1, \frac{c_{11}}{c_{3}}-c_{10}-1\right)}\left(1-2 c_{3} s\right)
\end{gathered}
$$

where,

$$
\begin{gathered}
c_{4}=\frac{1}{2}\left(1-c_{1}\right), c_{5}=\frac{1}{2}\left(c_{2}-2 c_{3}\right),, \\
c_{6}=c_{5}^{2}+\xi_{1}, c_{7}=2 c_{4} c_{5}-\xi_{2}, c_{8}=c_{4}^{2}+\xi_{3}, \\
c_{9}=c_{3} c_{7}+c_{3}^{2} c_{8}+c_{6}, c_{10}=c_{1}+2 c_{4}+2 \sqrt{c_{8}}, \\
c_{11}=c_{2}-2 c_{5}+2\left(\sqrt{c_{9}}+c_{3} \sqrt{c_{8}}\right), \\
c_{12}=c_{4}+\sqrt{c_{8}}, c_{13}=c_{5}-\left(\sqrt{c_{9}}+c_{3} \sqrt{c_{8}}\right)
\end{gathered}
$$

and $P_{n}$ is the orthogonal Jacobi polynomial.

\section{Factorization Method}

The three dimensional relativistic Klein-Gordon equation with mixed vector and scalar central potentials is written as:

$$
\left[\nabla^{2}+(V(r)-E)^{2}-(S(r)+M)^{2}\right\rfloor \psi(r, \theta, \varphi)=0
$$

where, $M$ is the rest mass, $E$ is the relativistic energy, and $S(r)$ and $V(r)$ are the scalar and vector potentials respectively and $\nabla^{2}$ is the Laplace operator. In spherical coordinate, the Klein-Gordon equation for a particle in the present of generalized Hylleraas potential $V(r)$ becomes: 


$$
\left[\begin{array}{l}
\frac{1}{r^{2}} \frac{\partial}{\partial r}\left(r^{2} \frac{\partial}{\partial r}\right)+\frac{1}{r^{2} \sin \theta} \frac{\partial}{\partial \theta}\left(\sin \theta \frac{\partial}{\partial \theta}\right)+\frac{1}{r^{2} \sin ^{2} \theta} \frac{\partial^{2}}{\partial \varphi^{2}} \\
-2(E V(r)+M S(r)) \\
+V^{2}(r)-S^{2}(r)+E^{2}-M^{2}
\end{array}\right] \psi(r, \theta, \varphi)=0 .
$$

The total wave function in Eq. (10) can be defined as

$$
\psi(r, \theta, \varphi)=\frac{R(r)}{r} Y(\theta, \varphi)
$$

and by decomposing the spherical wave function in Eq. (10) using Eq. (11) and the potential $V(r)$ in Eq. (1) for special case of equal scalar and vector potential (i.e $V(r)=S(r)$, we obtain the following equations:

$$
\begin{gathered}
\frac{d^{2} R(r)}{d r^{2}}+ \\
{\left[E^{2}-M^{2}+2(E+M) V(r)-\frac{\lambda}{r^{2}}\right] R(r)=0} \\
\frac{1}{\sin \theta} \frac{d}{d \theta}\left(\sin \theta \frac{d \Theta(\theta)}{d \theta}\right)+\left(\lambda-\frac{m^{2}}{\sin ^{2} \theta}\right) \Theta(\theta)=0 \\
\frac{d^{2} \Phi(\varphi)}{d \varphi^{2}}+m^{2} \Phi(\varphi)=0
\end{gathered}
$$

where, $\lambda=l(l+1)$ and $m^{2}$ are the separation constants. The solution of Eqs. (13) and (14) are well known [33]. Eq. (12) is the radial of Klein-Gordon equation respectively which is subject for discussion in the preceding section.

\section{Solutions of the Radial Klein-Gordon Equation}

For eigenvalues and corresponding eigen functions of the radial part of the Klein-Gordon equation, we substitute Eq. (1) into Eq. (12) to obtain:

$$
\begin{gathered}
\frac{d^{2} R(r)}{d r^{2}}+\left[E^{2}-M^{2}+2(E+M)\left(V_{1} \frac{a+e^{\alpha r}}{b+e^{\alpha r}}-\right.\right. \\
\left.\left.V_{2} \frac{d+e^{\alpha r}}{b+e^{\alpha r}}\right)-\frac{\lambda}{r^{2}}\right] R(r)=0
\end{gathered}
$$

The considered position dependent mass in this case is defined as:

$$
M(r)=M_{0}+\frac{M_{1}}{1+b e^{-\alpha r}}
$$

where, $M_{0}$ and $M_{1}$ are constant masses. The most extensive use of such kind of mass is in the physics of semiconductor quantum well structures [34]. If one set $M_{0}=0$, the position dependent mass is in agreement with that of Ref. [29].

Eq. (15) has no analytical or exact solution for $l \neq 0$ due to the centrifugal term, but can be solved approximately. Here we make use of an approximation scheme defined as:

$$
\frac{1}{r^{2}} \approx \frac{4 \alpha^{2}}{\left(1+b e^{-\alpha r}\right)^{2}}
$$

which is valid for $\alpha r \leq 1$ when $b=-1$, which is similar to other related work [3]. In addition, when performing a power series expansion and setting $\alpha \rightarrow 0$, Eq. (17) gives the desired $r^{-2}$ suggested by Greene and Aldrich [17]. In order to test accuracy of our scheme, we have compared the approximation of Eq. (17) for $\alpha=0.1$ denoted as f2 for $b=-1$ with the centrifugal term $\mathrm{f} 1=\frac{1}{r^{2}}$ in Fig. 1. This shows that the approximation is in good agreement with the centrifugal term.

Substituting Eqs. (16) and (17) into Eq. (15) and in order to reduce the resulting equation into Nikiforov-Uvarov equation, we make the transformation $S=e^{\alpha r}$; thus we have our desired equation as: 


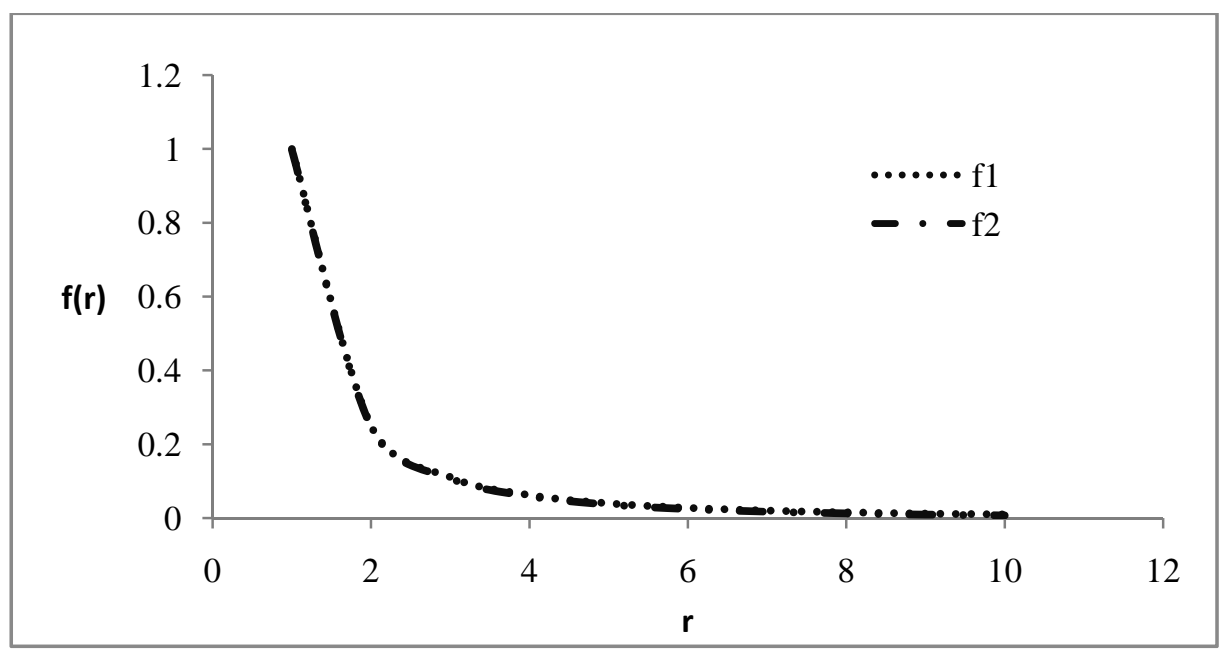

Fig. 1 Comparison of the centrifugal term $\mathbf{f} 1=\frac{1}{r^{2}}$ with the approximation $\mathbf{f} 2$ for $\alpha=0.05$ for $b=-1$.

$$
\frac{d^{2} R(s)}{d s^{2}}+\frac{\left(1+\frac{1}{b} s\right)}{s\left(1+\frac{1}{b} s\right)} \frac{d R(s)}{d s}+\frac{1}{s^{2}\left(1+\frac{1}{b} s\right)^{2}}\left[-Q_{1} s^{2}+Q_{2} s-Q_{3}\right] R(s)=0
$$

where the following dimensionless quantities have been defined as:

$$
\begin{gathered}
-\varepsilon^{2}=\frac{E^{2}-M_{0}^{2}}{\alpha^{2}} \\
Q_{1}=\varepsilon^{2}+\frac{2 M_{0} M_{1}}{\alpha^{2}}+\frac{M_{1}^{2}}{\alpha^{2}}+\frac{2 M_{1}\left(V_{2}-V_{1}\right)}{\alpha^{2}}+\frac{2\left(E+M_{0}\right)\left(V_{2}-V_{1}\right)}{\alpha^{2}}+4 \lambda \\
Q_{2}=-2 \varepsilon^{2}-\frac{2 b M_{0} M_{1}}{\alpha^{2}}+\frac{2(a+b)\left(E+M_{0}\right) V_{1}}{\alpha^{2}}-\frac{2(d+b)\left(E+M_{0}\right)}{\alpha^{2}}+\frac{2 M_{1}\left(a V_{1}-d V_{2}\right)}{\alpha^{2}} \\
Q_{3}=b^{2} \varepsilon^{2}-\frac{2 a b\left(E+M_{0}\right) V_{1}}{\alpha^{2}}+\frac{2 b d\left(E+M_{0}\right) V_{2}}{\alpha^{2}}
\end{gathered}
$$

Comparing Eq. (18) with Eq. (4) and making use of Eq. (8), we obtain the following parameters:

$$
\begin{aligned}
& c_{1}=1, c_{2}=c_{3}=-\frac{1}{b}, \\
& \xi_{1}=Q_{1}=\varepsilon^{2}+\frac{2 M_{0} M_{1}}{\alpha^{2}}+\frac{M_{1}^{2}}{\alpha^{2}}+\frac{2 M_{1}\left(V_{2}-V_{1}\right)}{\alpha^{2}}+\frac{2\left(E+M_{0}\right)\left(V_{2}-V_{1}\right)}{\alpha^{2}}+4 \lambda, \\
& \xi_{2}=Q_{2}=-2 b \varepsilon^{2}-\frac{2 b M_{0} M_{1}}{\alpha^{2}}+\frac{2(a+b)\left(E+M_{0}\right) V_{1}}{\alpha^{2}}-\frac{2(d+b)\left(E+M_{0}\right) V_{2}}{\alpha^{2}}+\frac{2 M_{1}\left(a V_{1}-d V_{2}\right)}{\alpha^{2}}, \\
& \xi=Q_{3}=b^{2} \varepsilon^{2}-\frac{2 a b\left(E+M_{0}\right) V_{1}}{\alpha^{2}}+\frac{2 d b\left(E+M_{0}\right) V_{2}}{\alpha^{2}}, \frac{1}{4 b^{2}}+\varepsilon^{2}+\frac{2 M_{0} M_{1}}{\alpha^{2}}+\frac{M_{1}^{2}}{\alpha^{2}}+\frac{2 M_{1}\left(V_{2}-V_{1}\right)}{\alpha^{2}}+\frac{2\left(E+M_{0}\right)\left(V_{2}-V_{1}\right)}{\alpha^{2}}+4 \lambda, \\
& c_{4}=0, c_{5}=\frac{1}{2 b}, c_{6}=\frac{2(a)\left(E+M_{0}\right) V_{2}}{\alpha^{2}}-\frac{2 M_{1}\left(a V_{1}-d V_{2}\right)}{\alpha^{2}},
\end{aligned}
$$




$$
\begin{aligned}
& c_{8}=b \varepsilon^{2}-\frac{2 a b\left(E+M_{0}\right) V_{1}}{\alpha^{2}}+\frac{2 d b\left(E+M_{0}\right) V_{2}}{\alpha^{2}}, c_{9}=\frac{1}{4 b^{2}}+\frac{2 M_{1}\left(a V_{1}-d V_{2}\right)}{b \alpha^{2}}+\frac{M_{1}^{2}}{\alpha^{2}}+\frac{2 M_{1}\left(V_{2}-V_{1}\right)}{\alpha^{2}}+4 \lambda \text {, } \\
& c_{10}=1+2 \sqrt{b^{2} \varepsilon^{2}-\frac{2 a b\left(E+M_{0}\right) V_{1}}{\alpha^{2}}+\frac{2 d b\left(E+M_{0}\right)}{\alpha^{2}}} \\
& c_{11}=-\frac{2}{b}+2\left(\begin{array}{l}
\sqrt{\frac{1}{4 b^{2}}+\frac{2 M_{1}\left(a V_{1}-d V_{2}\right)}{b \alpha^{2}}+\frac{M_{1}^{2}}{\alpha^{2}}+\frac{2 M_{1}\left(V_{2}-V_{1}\right)}{\alpha^{2}}+4 \lambda} \\
-\frac{1}{b} \sqrt{b^{2} \varepsilon^{2}-\frac{2 a b\left(E+M_{0}\right) V_{1}}{\alpha^{2}}+\frac{2 d b\left(E+M_{0}\right) V_{2}}{\alpha^{2}}}
\end{array}\right), \\
& c_{12}=\sqrt{b^{2} \varepsilon^{2}-\frac{2 a b\left(E+M_{0}\right) V_{1}}{\alpha^{2}}+\frac{2 d b\left(E+M_{0}\right) V_{2}}{\alpha^{2}}}, \\
& c_{13}=\frac{1}{2 b}-\left(\begin{array}{l}
\sqrt{\frac{1}{4 b^{2}}+\frac{2 M_{1}\left(a V_{1}-d V_{2}\right)}{b \alpha^{2}}+\frac{M_{1}^{2}}{\alpha^{2}}+\frac{2 M_{1}\left(V_{2}-V_{1}\right)}{\alpha^{2}}+4 \lambda} \\
-\frac{1}{b} \sqrt{b^{2} \varepsilon^{2}-\frac{2 a b\left(E+M_{0}\right) V_{1}}{\alpha^{2}}+\frac{2 d b\left(E+M_{0}\right) V_{2}}{\alpha^{2}}}
\end{array}\right) .
\end{aligned}
$$

Substituting Eqs. (19)-(23) into Eq. (6), we obtain the energy eigenvalues equation for GHP with position dependent mass as:

$$
\begin{aligned}
& -\frac{n^{2}}{b}-\frac{1}{2 b}(2 n+1)+(2 n+1)\left(\begin{array}{l}
\sqrt{\frac{1}{4 b^{2}}+\frac{2 M_{1}\left(a V_{1}-d V_{2}\right)}{b \alpha^{2}}+\frac{M_{1}^{2}}{\alpha^{2}}+\frac{2 M_{1}\left(V_{2}-V_{1}\right)}{\alpha^{2}}+4 \lambda} \\
-\frac{1}{b} \sqrt{b^{2} \varepsilon^{2}-\frac{2 a b\left(E+M_{0}\right) V_{1}}{\alpha^{2}}+\frac{2 d b\left(E+M_{0}\right) V_{2}}{\alpha^{2}}}
\end{array}\right)+\frac{2 b M_{0} M_{1}}{\alpha^{2}} \\
& -\frac{2(a+b)\left(E+M_{0}\right) V_{1}}{\alpha^{2}}+\frac{2(d+b)\left(E+M_{0}\right) V_{2}}{\alpha^{2}}-\frac{2 M_{1}\left(a V_{1}-d V_{2}\right)}{\alpha^{2}}+\frac{2 a\left(E+M_{0}\right) V_{1}}{\alpha^{2}}
\end{aligned}
$$

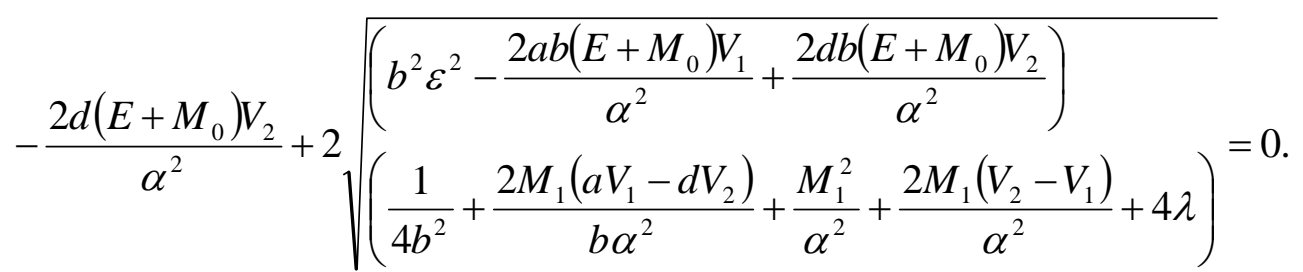

Solving Eq. (24) explicitly, we obtain the energy eigenvalues for the radial part of the Klein-Gordon equation for equal scalar and vector GHP with position dependent mass as

$$
E^{2}-M_{0}^{2}=-\frac{1}{4 b^{2}}\left[\frac{A-4 b \lambda+\left(n+\frac{1}{2}-\delta\right)^{2}}{\left(n+\frac{1}{2}-\delta\right)}\right]^{2}+\frac{d\left(E+M_{0}\right) V_{2}}{2 b}-\frac{a\left(E+M_{0}\right) V_{1}}{2 b},
$$

where, $A=-\frac{M_{1} b}{\alpha^{2}}-\frac{2 b M_{1}\left(V_{2}-V_{1}\right)}{\alpha^{2}}-\frac{2 M_{0} M_{1} b}{\alpha^{2}}+\frac{2 b\left(E+M_{0}\right) V_{1}}{\alpha^{2}}-\frac{2 b\left(E+M_{0}\right) V_{2}}{\alpha^{2}}$,

and

$$
\delta=\left(\sqrt{\frac{1}{4}+\frac{2 M_{1} b\left(a V_{1}-d V_{2}\right)}{\alpha^{2}}+\frac{M_{1}^{2} b^{2}}{\alpha^{2}}+\frac{2 M_{1} b^{2}\left(V_{2}-V_{1}\right)}{\alpha^{2}}+4 b^{2} \lambda}\right)
$$


Using Eqs. (7) and (23), corresponding wave function of the radial part is obtained as:

where,

$$
R(s)=N_{n l} s^{\mu}\left(1+\frac{1}{b} s\right)^{\frac{1}{2}+v} P_{n}^{(2 \mu, 2 v)}\left(1+\frac{1}{b} 2 s\right),
$$

$$
\begin{gathered}
v=-b\left(\sqrt{\frac{1}{4 b^{2}}+\frac{2 M_{1}\left(a V_{1}-d V_{2}\right)}{b \alpha^{2}}+\frac{M_{1}^{2}}{\alpha^{2}}+\frac{2 M_{1}\left(V_{2}-V_{1}\right)}{\alpha^{2}}+4 \lambda}\right), \\
\mu=\sqrt{b^{2} \varepsilon^{2}-\frac{2 a b\left(E+M_{0}\right) V_{1}}{\alpha^{2}}+\frac{2 d b\left(E+M_{0}\right) V_{2}}{\alpha^{2}}} .
\end{gathered}
$$

Using the transformation, $s=e^{\alpha r}$, Eq. (26) can also be written as:

$$
R(r)=N_{n l} e^{\alpha r}\left(1+\frac{1}{b} e^{\alpha r}\right)^{\frac{1}{2}+v} P_{n}^{(2 \mu, 2 v)}\left(1+\frac{1}{b} 2 e^{\alpha r}\right)
$$

where, $N_{n l}$ is a normalization constant.

\section{A Few Limiting Cases}

Let us now study some limiting cases of this potential under investigation. Some well known potentials in the literature are obtained by choosing appropriate parameters in the generalized Hylleraas potential. A few of such potentials are discussed below:

\subsection{Rosen-Morse Potential}

By setting $V_{1}=V_{0}, a=1, b=-1, d=0, V_{2}=0$ and mapping $\alpha \rightarrow-2 \alpha$ in Eq. (1) reduces the generalized Hylleraas potential into Rosen-Morse potential [35] of the form:

$$
V(r)=-\frac{V_{0}\left(1+e^{-2 \alpha r}\right)}{\left(1-e^{-2 \alpha r}\right)}
$$

and corresponding energy is obtained by substituting the above parameters into the energy eigenvalues of Eq. (25).

\subsection{Woods-Saxon Potential}

$$
\text { Choosing } \quad a=d=V_{2}=0, b=1, V_{1}=-V_{0}
$$
reduces the potential of Eq. (1) into Woods-Saxon potential [36]

$$
V(r)=-\frac{V_{0} e^{\alpha r}}{\left(1+e^{\alpha r}\right)}
$$

Whose energy is obtained by substituting the above chosen potential parameters into Eq. (25).

\subsection{Hulthen Potential}

Setting $\quad a=1+d, b=-1, V_{2}=V_{1}=V_{0} \quad$ and mapping $\alpha \rightarrow 2 \alpha$ into Eq. (1), a well known Hulthen potential is obtained as a result [37]:

$$
V(r)=-\frac{V_{0} e^{-2 \alpha r}}{1-e^{-2 \alpha r}}
$$

The corresponding energy for this Hulthen potential is obtained by substituting the set potential parameters into the energy spectrum in Eq. (25).

\section{Conclusions}

In this paper, we have obtained the approximate bound state solutions of the relativistic Klein-Gordon equation in the case of equal scalar and vector generalized Hylleraas potential with position dependent mass using parametric form of Nikiforov-Uvarov method with the help of approximation scheme to evaluate the centrifugal term. The bound states energy eigenvalues and the corresponding wave functions in terms of Jacobi polynomial are obtained. Our results could be used to study the interactions and binding energies of the central potential for diatomic molecules in the relativistic framework. The results will also have many applications in chemical and molecular physics and the recently reported result of neutron-proton pairs in heavy nuclei using perturbation theory [38]. Also, this 
problem under investigation will have great applications in the nonrelativistic quantum mechanics in the limiting cases as reported in recent works [39-41]. By appropriate choice of potential parameters our potential in Eq. (1) reduces to a few well known potentials in the literature.

\section{References}

[1] Dirac, P. A. 1958. The Principles of Quantum Mechanics. Oxford University Press.

[2] Landau, L. D., and Lifshitz, E. M. 1977. Quantum Mechanics, Non-Relativistic Theory. 3rd Edition, Oxford, UK: Pergamon.

[3] Greiner, W. 2000. Relativistic Quantum Mechanics: Wave equation. Berlin: Springer.

[4] Yi, L. Z., Diao, Y. F., Liu, J. Y., and Jia, C. S. 2004. "Bound States of the Klein-Gordon Equation with Vector and Scalar Rosen-Morse-Type Potentials.” Phys. Lett. A 333: 212-7.

[5] Ikot, A. N. 2011. Afri. Rev. Phys. 6 (0026): 221.

[6] Antia, A. D., Essien, I. E., Umoren, E. B., and Eze, C. C. 2015. Advances in Physics Theories and Applications 44: 1-13.

[7] Antia, A. D., Akpan, I. O., and Akankpo, A. O. 2015. International Journal of High Energy Physics 2 (4): 50-5.

[8] Ikot, A. N., Awoga, O. A., Antia, A. D., Hassanabadi, H., and Maghsoodi, E. 2013. "Approximate Solutions of D-Dimensional Klein-Gordon Equation with Modified Hylleraas Potential.” Few-Body Systems 54 (11): 2041-51.

[9] Hassanabadi, H., Zarrinkamar, S., and Rajabi, A. A. 2011. "Exact Solutions of D-Dimensional Schroedinger Equation for an Energy-Dependent Potential by NU Method.” Commun. Theor. Phys. 55: 541-5.

[10] Qiang, W. C., and Dong S. H. 2009. Phys. Scr. 79: 045004.

[11] Antia, A. D., Ikot, A. N., Hassanabadi, H., and Maghsoodi, E. 2013. Indian Journal of Physics 87 (11): 1133-9.

[12] Wei, G. F., Long, C. Y., Duan, X. Y., and Dong, S. H. 2008. Phys. Scr. 77: 035001.

[13] Dong, S., Garcia-Ravalo, J., and Dong, S.-H. 2007. "Analytical Approximations to the l-Wave Solutions of the Schrödinger Equation with an Exponential-Type Potential.” Phys. Scr. 76 (4): 393.

[14] Oyewumi, K. J., and Akoshile, C. O. 2010. Eur. Phys. J. A 45: 311-8.

[15] Antia, A. D., Ikot, A. N., Ituen, E. E., and Akpan, I. O. 2012. Sri Lankan J. Phys. 13 (1): 27-40.

[16] Lu, J. 2005. “Analytic Quantum Mechanics of Diatomic Molecules with Empirical Potentials.” Phys. Scr. 72 (5): 349.

[17] Greene, R. L., and Aldrich, C. 1976. Phys. Rev. A 14:
2363.

[18] Jia, C. S., Chen, T., and Cui, L. G. 2009. Phys. Lett. A 373: 1621.

[19] Hill, E. H. 1954. Am. J. Phys. 22: 1712.

[20] Yasuk, F., and Bahar M. K. 2012. Phys. Scr. 85: 045004.

[21] Hassanabadi, H., Maghsoodi, E., and Zarrinkamar, S. 2012. Euro. Phys. J. Plus 127: 31.

[22] Mustafa, O., and Sever, R. 1991. Phys. Rev. A 44: 4142.

[23] Antia, A. D., Ituen, E. E., Obong, H. P., and Isonguyo, C. N. 2015. Int. J. Recent advances in Phys. 4 (1): 55-65.

[24] Ikhdair, S. M., and Sever, R. 2008. Int. J. Mod. Phys. 19: 221-9.

[25] Nikiforov, A. F., and Uvarov, V. B. 1988. Special Functions of Mathematical Physics. Basel: Birkhauser.

[26] Ikot, A. N. 2012. Chin. Phys. Lett. 29: 060307.

[27] Hassanabadi, H., Yazarlo, B. H., Zarrinkamar, S., and Rajabi, A. A. 2011. Phys. Rev. C 84: 064003.

[28] Dong, S. H., and Lozaada-Cassou, M. 2006. Phys. Scr. 74 (2): 285-7.

[29] Meyur, S., Maji, S., and Debrath, S. 2014. "Analytical Solution of the Schrödinger Equation with Spatially Varying Effective Mass for Generalised Hylleraas Potential.” Advances in High Energy Physics 952597.

[30] Hylleraas, E. A. 1935. J. Chem. Phys. 3 (9): 595.

[31] Ikot, A. N. 2013. Comm. Theor. Phys. 59 (3): 268-73.

[32] Tezcan, C., and Sever, R. 2009. "A General Approach for the Exact Solution of the Schrödinger Equation.” Int. J. Theor. Phys. 48 (2): 337-50.

[33] Schiff, L. I. 1955. Quantum Mechanics. New York, McGraw Hill.

[34] Gonul, B., Tutcu, D., and Ozer, O. 2002. Modern Physics letters A 17 (31): 2057-66.

[35] Debnath, S., and Biswas, B. 2012. “Analytical Solutions of the Klein-Gordon Equation for Rosen-Morse Potential via Asymptotic Iteration Method.” EJTP 26: 191-8.

[36] Ikot, A. N., Akpabio, L. E., and Obu, J. A. 2011. J. Vect. Relat. 6 (1): 1.

[37] Ikot, A. N., Akpabio, L. E., and Uwah, E. J. 2011. "Bound State Solution of the Klein-Gordon Equation with Hulthen Potential.” Elect. J. Theor. 8 (25): 225-32.

[38] Khanna, K. M., Kanyeki, G. F., Rotich, S. K., Torongey, P. K., and Ameka, S. E. 2010. Indian J. Pure and Appl. Phys. 48: 7.

[39] Azimzadeh, Z., Vahidi, A. R., and Babolian, E. 2012. "Exact Solutions for Non-Linear Duffing's Equations by He’s Homotopy Perturbation Method.” Indian J. Phys. 86: 721-6.

[40] Biswas, A., and Krishnan, E. 2011. Indian J. Phys. 85 (10): 1513-21.

[41] Wamalwa, D. S., and Omolo, J. A. 2010. Indian J. Phys. 84: 1241 